Femmes africaines séropositives en quête d'asile. Opportunités et contraintes de la politique migratoire canadienne

HIV-positive African Women in Search of Asylum. Opportunities and Constraints of the Canadian Migratory Policy

Mujeres africanas seropositivas en busca de asilo. Oportunidades y limitaciones de la política migratoria canadiense

\title{
Émilie Adam-Vézina
}

\section{(2) OpenEdition}

\section{Journals}

Édition électronique

URL : https://journals.openedition.org/remi/5931

DOI : 10.4000/remi.5931

ISSN : $1777-5418$

\section{Éditeur}

Université de Poitiers

\section{Édition imprimée}

Date de publication : 1 juin 2012

Pagination : 129-148

ISBN : 979-10-90426-04-7

ISSN : 0765-0752

\section{Référence électronique}

Émilie Adam-Vézina, «Femmes africaines séropositives en quête d'asile. Opportunités et contraintes de la politique migratoire canadienne », Revue européenne des migrations internationales [En ligne], vol. 28 - n² | 2012, mis en ligne le 10 octobre 2012, consulté le 14 avril 2022. URL : http:// journals.openedition.org/remi/5931; DOI : https://doi.org/10.4000/remi.5931 


\section{Femmes africaines séropositives en quête d'asile. Opportunités et contraintes de la politique migratoire canadienne}

\section{Émilie ADAM-VÉZINA ${ }^{1}$}

T'état de santé des populations migrantes a fait l'objet d'études importantes développement de modèles explicatifs d'une bonne ou mauvaise condition de santé. Les données statistiques canadiennes sur la santé des nouveaux arrivants montrent que les migrants et non migrants présentent un état de santé similaire (Chen et Wilkins, 1996 ; Laroche, 2000 ; McDonald et Kennedy, 2004 ; Newbold et Danforth, 2003), voire même une meilleure santé chez les premiers, surtout s'ils sont des migrants récents (Ali et al., 2004), un phénomène mieux connu sous le nom d'effet «immigrant en bonne santé » ( "healthy immigrant effect»). Une partie importante de la littérature canadienne sur la santé des nouveaux arrivants s'est intéressée à ce phénomène pour en conclure que ce meilleur état de santé résultait d'une double sélection. D'abord par le fait que les individus candidats à la migration sont habituellement ceux en bonne forme physique et mentale. Ensuite en raison des politiques migratoires canadiennes qui privilégient les migrants en bonne condition de santé. Les recherches montrent toutefois que cet effet diminue ou s'annule au fil du temps de résidence au Canada (Ali et al., 2004 ; De Maio, 2010 ; McDonald et Kennedy 2004 ; Newbold et al., 2003). Certains déterminants de la santé des migrants, tels que le genre, le statut socio-économique, l'accès aux soins ou les discriminations qui accompagnent l'installation dans ce pays ont pu être explorés, pour expliquer leur poids dans la dégradation de l'état de santé (Cognet, 2004 ; De Maio, 2010 ; De Maio et Kemp, 2010 ; Hyman, 2009 ; Newbold et Danforth, 2003)².

Ces études fournissent un socle de connaissances solide, mais ne rendent pas bien compte des différences sanitaires entre les divers groupes de nouveaux arrivants. Ainsi, « l'effet de la bonne santé des migrants », constaté principalement chez les migrants économiques ou issus du regroupement familial, masque l'état de santé d'autres groupes comme les réfugiés, qui tend à être plus mauvais à l'arrivée sur le territoire canadien

1 Doctorante socio-anthropologie, Université Paris Diderot, Laboratoire URMIS (UMR IRD 205), case courrier 7027, 75205 Paris cedex 13 ; emilie.adam@etu.univ-paris-diderot.fr

2 Sur cette question, voir l'article de Cognet et Hamel dans ce numéro. 
(McKeary et Newbold, 2010 ; Oxman-Martinez et al., 2005 ; Zhao et Gilkinson, 2010). Bien qu'il soit possible de présumer que cette santé dégradée découle des motifs qui poussent à l'exil, nous connaissons encore peu les trajectoires migratoires de ces populations et moins encore la manière dont les politiques migratoires canadiennes encadrent et conditionnent l'expérience migratoire des réfugiés malades. Le champ des études sur la santé des réfugiés au Canada demeure lacunaire bien que certaines dimensions aient été traitées comme la santé mentale (Beiser et al., 1998, Kirmayer et al., 2011 ; Moreau et al., 1999) ou l'accès aux soins et services de santé (Gagnon, 2002 ; Rousseau et al., 2008).

En 2001, au Canada, les politiques d'immigration ont été modifiées, introduisant des critères spécifiques et contraignants pour l'entrée et le séjour des migrants malades. Ce contexte politique nous conduit ici à interroger l'impact des politiques migratoires et sanitaires canadiennes, sur les trajectoires migratoires ${ }^{3}$ de femmes séropositives africaines qui ont migré vers ce pays. Dans un premier temps, bien que la législation régissant l'entrée et le séjour des étrangers soit un sujet complexe, nous en présenterons le cadre principal. Puis, après avoir campé les grandes lignes de ce dispositif législatif et réglementaire, nous nous appuierons sur les récits des femmes rencontrées dans le cadre d'un terrain mené en 2009-2010, pour montrer comment il influence leurs trajectoires migratoires et de soins.

\section{LES POLITIQUES MIGRATOIRES CANADIENNES}

Le Canada s'est construit en grande partie sur diverses vagues d'immigration et compte aujourd'hui un résident sur six né à l'étranger ${ }^{4}$. Les politiques migratoires actuelles pourraient s'apparenter à celles d'une immigration « choisie », c'est-à-dire basée sur des quotas que le gouvernement fédéral fixe pour des catégories d'entrée privilégiées (principalement liées aux besoins de main-d'œuvre pour certains secteurs d'activité). Cette sélection, qui compte sur les migrants comme force de travail, requiert que les postulante-s soient en bonne santé.

Votée le $1^{\text {er }}$ novembre 2001 par le Parlement du Canada et entrée en vigueur le 28 juin 2002, la Loi sur l'immigration et la protection des réfugiés (LIPR) constitue le document législatif qui régit les conditions d'entrée et de séjour des étrangers dont le Règlement sur l'immigration et la protection des réfugiés définit les modalités d'application.

La situation géographique du pays, avec pour seule frontière terrestre les ÉtatsUnis, n'en fait pas une destination aisément accessible. Pour entrer légalement au Canada, une personne peut faire une demande de titre de séjour (court et long séjours) à partir de son pays de départ ou se présenter à la frontière pour déposer une demande d'asile. Les catégories d'entrée se répartissent en deux grands types de statuts : les demandes de séjour

3 Nous utilisons le terme pour faire apparaitre l'expérience migratoire dans sa globalité : l'entrée par trajectoire migratoire permet de saisir la complexité des motifs d'émigration et d'analyser conjointement les mouvements de mobilité dans l'espace des migrants sans négliger les politiques qui les structurent (Guillou, 2009 ; Jolivet, 2007).

4 Citoyenneté et Immigration Canada (CIC), http://www.cic.gc.ca/francais/index.asp 
temporaire (qui ouvrent sur un statut de « résidents temporaires » dans la loi canadienne) et les demandes de séjour permanent (les « résidents permanents $\left.{ }^{5} »\right)$.

Certaines conditions sont exigées pour entrer dans le pays. Dans le cas des demandes de résidence d'une durée inférieure à six mois, il est exigé un visa de résidence temporaire sauf pour les personnes venant de pays qui en sont dispensés ${ }^{6}$. Selon le type de visa demandé (visiteur, étudiant et travailleur temporaire), des pièces spécifiques sont requises ${ }^{7}$. Les demandes se font généralement à l'extérieur du Canada via un bureau canadien des visas dans leur pays d'origine ou dans un pays limitrophe.

Certaines exigences spécifiques sont associées à chaque catégorie de résidence temporaire et, sans en faire l'inventaire exhaustif, nous souhaitons souligner quatre aspects importants qui encadrent l'attribution de la résidence temporaire et qui ont un impact sur les choix effectués par les migrant-e-s :

1) Le requérant-e doit « convaincre ${ }^{8}$ les agents de sa « bonne foi » quant à son engagement à quitter le pays lorsque le titre de séjour sera arrivé à son terme ;

2) Le requérant-e doit prouver qu'il ou elle dispose de ressources financières suffisantes durant son séjour ;

3) Le requérant-e doit « être en bonne santé »" , un certificat médical peut être demandé pour le prouver ;

4) Le requérant-e ne doit pas constituer une menace pour la sécurité, un certificat de police peut être demandé.

Le candidat à l'immigration doit donc prouver, à la fois, que son objectif est un séjour d'une durée limitée dans le pays et, à la fois, qu'il ne constitue pas une menace pour la santé et la sécurité de la population canadienne. Les motifs sanitaires contenus dans la loi peuvent conduire à la demande d'un examen médical - y compris pour les personnes exemptes de visa temporaire - sans que celui-ci soit automatique (il est laissé au pouvoir discrétionnaire des agents instruisant la demande).

Si le statut de résident permanent est sollicité depuis un pays étranger, les conditions d'accès au territoire canadien sont sensiblement identiques à celles exigées pour un séjour temporaire, avec cependant un contrôle plus strict des pièces à fournir pour authentifier sa situation financière, sanitaire, sécuritaire, etc. Les requérants doivent également satisfaire aux critères (âge, niveau de scolarité, connaissance des langues officielles,

5 Il est nécessaire de clarifier ce que le terme de « résidence permanente » signifie dans la loi canadienne. Ce statut juridique offre une stabilité de séjour sans besoin de renouvellement, mais n'octroie pas la totalité des droits dont bénéficie le citoyen canadien.

6 Un nombre important de pays sont listés dont la France, les États-Unis, la Grande-Bretagne ainsi que plusieurs pays européens. Hormis la Namibie et le Botswana, les pays du continent africain ne font pas l'objet de dispense de visa (CIC, http://www.cic.gc.ca/francais/visiter/visas. asp\#dispenses). Pour les personnes dispensées, le passeport est néanmoins obligatoire.

7 Ainsi une demande de visa de résident temporaire à titre étudiant, requiert la preuve d'inscription dans un établissement d'enseignement canadien ; pour les travailleurs temporaires une promesse d'embauche.

8 Terme employé par Citoyenneté et Immigration Canada (CIC) sur son site internet : « convaincre un agent d'immigration » (http://www.cic.gc.ca/francais/visiter/demande-qui.asp).

9 http://www.cic.gc.ca/francais/visiter/demande-qui.asp 
expériences, etc.) conformément au système en vigueur depuis 1967 (Weibe, 2009). Si le demandeur répond aux critères déterminés, il sera obligatoirement soumis à un examen clinique général, physique et mental, comprenant, entre autres, une radiographie pulmonaire de dépistage de la tuberculose et des analyses médicales incluant une sérologie VIH. La législation actuelle stipule qu'un candidat à l'immigration, que ce soit pour un visa temporaire ou permanent, peut être déclaré inadmissible pour raisons médicales et se voir refuser l'entrée au Canada ou l'octroi d'un titre de séjour :

1) s'il peut représenter un danger pour la santé ou la sécurité publique ;

2) s'il risque d'entrainer un «fardeau excessif $»{ }^{10}$ pour les services sociaux ou de santé du Canada.

Un mauvais état de santé et ses possibles conséquences en termes de besoins de soins, de services sociaux, comme celles qui relèveraient d'une incapacité à travailler et à subvenir à ses besoins essentiels, entraînent généralement un refus de la demande, en raison du risque de "fardeau excessif pour les services sociaux ou de santé au Canada ${ }^{11}$ encouru, sauf pour certaines catégories de migrants, dont les demandeurs d'asile.

Or, si depuis 1991, une personne séropositive n'est plus considérée comme une "menace pour la santé et la sécurité publiques », elle demeure soumise à la clause du «fardeau excessif» pour les services de santé et services sociaux canadiens. La loi d'immigration canadienne de 2001 clarifie cette clause pour le VIH, demeurée jusqu'alors peu précise : dorénavant, le dépistage de la maladie est obligatoire pour tous les demandeurs d'un visa permanent. Les demandes qui proviennent de personnes séropositives prenant des antirétroviraux (ARV) - dont nous rappelons qu'elles ne sont pas systématiquement en état de sida avéré et que, parce qu'elles peuvent aujourd'hui vivre de longues années avec une charge virale indétectable et sans développer de maladies, sont tout à fait en état de travailler et de conduire leurs affaires comme toute autre personne - sont quasi systématiquement rejetées, car « inadmissibles pour raisons médicales » (Réseau juridique canadien VIH/sida, 2011 : 5). De leur côté, les personnes séropositives en bonne santé et qui ne prennent pas de traitement ARV voient généralement leur demande acceptée, si l'évaluation que fait Citoyenneté et Immigration Canada (CIC) par une anticipation des coûts sociaux et sanitaires de leur condition, ne les classe pas comme des futurs «fardeaux excessifs » pour le système de santé.

Dans le cas des demandes d'asile, l'article 97 (1) de la Loi sur l'immigration et la protection des réfugiés stipule que la demande ne peut résulter uniquement « de l'inca-

10 Une personne considérée comme « fardeau » est désignée comme : 1) une personne qui nécessite des soins et des traitements qui dépassent les dépenses de l'État pour un Canadien sur une année. Ce coût varie chaque année et est calculé à l'aide des données fournies par l'Institut canadien d'informations sur la santé. Lors de l'étude des dossiers des requérant-e-s, ce calcul estime, sur une période de cinq ans, le coût imposé au système sanitaire et social du Canada. Dans le cas de certaines maladies chroniques, dont le VIH, cette période peut être rallongée à dix ans (Réseau juridique canadien VIH/sida, 2009 : 2). 2) Une personne qui nécessite des soins qui sont susceptibles de « retarder » le fonctionnement du système de santé canadien et d'impliquer un délai dans l'accès aux soins (allongement des listes d'attente par exemple) pour les citoyens ou résidents canadiens avec le risque envisagé d'une augmentation des taux de morbidité et mortalité (Réseau juridique canadien VIH/sida, 2009 : 2).

$11 \mathrm{http}: / /$ www.cic.gc.ca/francais/information/medicaux/examen-medical-perm.asp 
pacité du pays de fournir des soins médicaux ou de santé adéquats ». Ainsi, une demande d'asile rejetée après toutes les procédures en appel peut conduire un étranger, même malade, à se voir reconduire à la frontière, sauf si son pays d'origine fait l'objet d'un moratoire ${ }^{12}$. Ainsi, " les personnes souffrant de troubles médicaux et qui risquent la mort, des sanctions excessives ou un traitement inhumain peuvent être autorisées à demeurer au Canada même si leur revendication du statut de réfugié échoue, mais seulement si le risque découle d'autre chose que du fait qu'elles ne peuvent pas recevoir des soins de santé adéquats dans leur pays d'origine » (Klein, 2001 : 27).

Au total, la loi canadienne n'exclut pas d'emblée tout migrant malade de l'entrée ou du séjour sur le territoire national. Une telle loi serait discriminatoire et donc contraire à la Charte canadienne des droits et libertés. Elle est cependant très restrictive pour les étrangers malades, sauf en cas de demande d'asile. Sur la base des chiffres fournis par CIC quant aux demandes d'immigration émanant de personnes séropositives par catégories statutaires entre 2002 et 2010, Bisaillon (2010 : 123) montre que les migrants temporaires ou économiques sont ceux dont la demande est la plus fréquemment rejetée. Les catégories avec les taux d'admissibilité les plus élevés sont les demandeurs d'asile et les migrants au titre du regroupement familial qui sont exempts de la contrainte du « fardeau médical ». La loi ne stipule pas que l'état de santé d'un étranger l'interdit d'entrée et de séjour, mais uniquement si, après une évaluation individualisée de sa demande, sa maladie risque de générer un coût financier trop élevé pour la société canadienne ou menacer la santé et la sécurité publiques.

Les politiques canadiennes d'accès au séjour sur le territoire national imposent ainsi des limites assez claires aux individus malades. Néanmoins, nous proposons de suivre, au travers de leurs parcours migratoires, des femmes africaines séropositives qui ont quitté leurs pays dans des conditions souvent difficiles - contextes de violences et de persécutions genrées - pour se mettre en quête d'un asile, de découvrir avec elles comment s'imposent les contraintes de la loi, quelles sont les opportunités qu'elles voient et quelles « stratégies » elles développent face à ces dispositifs.

\section{FUIR LES VIOLENCES, LES PARCOURS D'EXIL}

Nous avons rencontré seize femmes entre 2009 et 2010 à Montréal, par l'intermédiaire d'une association montréalaise active depuis 1990 dans le soutien et l'information aux femmes vivant avec le VIH-sida. Ces femmes proviennent de la région des Grands Lacs africains : la majorité du Burundi (douze), mais également du Rwanda (deux) et du Congo (deux). Après avoir été informées des objectifs de la recherche ${ }^{13}$ et des règles éthiques (confidentialité, données dénominalisées, enregistrement audio avec accord, etc.), les entretiens se sont déroulés dans le lieu de leur choix, souvent à leur domicile.

12 En 2011, il s'agissait de l'Afghanistan, Haïti, Irak, République démocratique du Congo, Zimbabwe (CCR, http://ccrweb.ca/).

13 Cette étude dont le titre provisoire est « Femmes malades, femmes en mouvement. Trajectoires migratoires et thérapeutiques de migrantes africaines en France et au Canada atteintes du $V I H$-sida » porte sur les trajectoires migratoires et en soins de femmes africaines séropositives en France et au Canada. Elle a reçu le soutien financier de Sidaction. 
L'idée de quitter le Rwanda et le Burundi elles l'ont envisagée, voire mise en œuvre, très tôt - notamment dès les massacres de 1993-1995. Certaines se sont, à l'époque, réfugiées temporairement au Kenya ou au Congo, mais, au fil des années, face au climat socio-politique instable de la région, la décision de s'exiler a mûri. Cependant, toutes le racontent, leur intention ou leur souhait de partir est déterminé par un événement décisif dans leur vie. Cette décision, ce choix du dernier espoir (Spijkerboer, 2000) qui confirme la nécessité de fuir, agit comme le révélateur d'une vie devenue insoutenable et elle n'est pas, loin s'en faut, strictement liée au fait d'être atteinte du VIH-sida bien qu'une majorité d'entre elles aient été informées de leur maladie avant leur départ.

Pour Françoise ${ }^{14}$, originaire du Burundi, c'est le viol commis sur sa fille puis la décision des autorités, malgré son dépôt de plainte, de ne pas arrêter l'agresseur, qui fondent sa décision. D'autant que ce dernier essaiera de la tuer et que, depuis plusieurs années, Françoise tentait de divorcer d'un mari qui contrôlait ses allées et venues, l'avait séquestrée et battue à plusieurs reprises. Suzanne, une autre Burundaise, s'efforçait elle aussi de fuir les violences infligées par son mari. Une rupture d'antirétroviraux, devenus indisponibles dans le pays, conduira à la chute de son système immunitaire et au développement de la maladie au stade du sida. À ce moment, elle raconte que sa vie est doublement menacée : par les coups de son mari et par la maladie.

Pour Françoise comme pour Suzanne et toutes les autres femmes interviewées, l'élément commun est le désir de sécurité pour elles, et le cas échéant pour leurs filles. Leurs vies sont menacées directement par un mari, des adversaires politiques ou des milices armées, mais également par une maladie qui nécessite des traitements dont l'approvisionnement est discontinu. Partir, oui, mais pour quel pays ? Et comment?

Le Canada s'est rapidement imposé comme la meilleure destination d'exil pour toutes nos interlocutrices. Certaines, en raison d'opportunités de travail ou de vacances, avaient découvert d'autres pays comme la France, la Belgique, l'Angleterre et les ÉtatsUnis sans arriver à s'y projeter pour vivre, à ce moment crucial de leur vie. Ce qui a emporté leur décision, ce sont les représentations - le plus souvent idéalisées - du Canada à l'étranger, celles d'un pays sûr, accueillant envers les étrangers et, qui plus est, un pays qui serait exempt de discrimination :

«Le Canada c'est un pays qui accueille les réfugiés et où il n'y a pas de discrimination. Pour les papiers et les démarches, c'est aussi plus facile qu'en Europe par exemple » (Antoinette, trente-six ans, réfugiée du Burundi, arrivée en 2007).

Le Canada est également une destination qu'elles ont choisie en raison de la pratique du français alors que peu d'entre elles maitrisent la langue anglaise. Elles ne distinguent pas pour autant la province de Québec et se figurent qu'elles pourront s'ex-

14 Les noms des femmes interviewées ont été modifiés afin de préserver leur anonymat. 
primer aisément en français dans tout le pays ${ }^{15}$. Elles se représentent également le Canada comme un pays où les droits des femmes sont respectés. Confrontées à l'impunité dont bénéficient les responsables des violences qu'elles ont subies ainsi que leurs proches, il leur importait avant tout de pouvoir espérer mener une nouvelle vie dans un contexte où les lois protègent les femmes :

« J'ai choisi le Canada parce que l'on parle français. Il y a aussi une femme de mon pays [le Burundi] qui vit ici et qui plaide pour les droits des femmes. Elle m'a dit qu'avec tous les problèmes que j'ai eus, il faut que je vienne au Canada. C'est un pays qui protège les femmes » (Suzanne, trente-quatre ans, réfugiée du Burundi, arrivée en 2008).

«Pourquoi le Canada? Parce que c'est un pays qui accepte les gens, qui les aide. Dans d'autres pays, j'ai vu que l'on ne faisait rien, surtout pour les femmes maltraitées et violées. J'ai souvent voyagé en Europe et je n'ai pas vu la même chose. Ici on t'écoute, on comprend que tu sois en danger. Ce n'est pas comme dans mon pays [le Burundi] où tu as toujours peur d'être tuée, où c'est l'impunité totale.

Tu ne sais pas où porter plainte et même si tu le fais, il ne se passe rien » (Louise, cinquante-six ans, réfugiée du Burundi, arrivée en 2008).

Toutes affirment avoir voulu entrer légalement au Canada, sans faire appel à des réseaux de passeurs. Tout ce qu'elles souhaitaient, c'était pouvoir accéder au territoire canadien pour y faire valoir la nécessité de leur protection. Pour ce faire, elles se sont informées, se sont renseignées sur les procédures, par Internet et auprès des ambassades ou des consulats, mais aussi auprès de leur entourage. Si elles étaient majoritaires à connaître des compatriotes aux États-Unis, très peu, en revanche, disent avoir été en contact avec des gens au Canada avant leur départ.

Très vite, elles se sont heurtées aux contraintes du système canadien d'immigration. Du côté de la demande d'asile, les chances étaient limitées : il leur aurait fallu soit quitter leur pays pour un autre pays africain où elles auraient pu déposer une demande ; soit se présenter à la frontière canadienne et, une fois sur place, demander l'asile ; soit se faire parrainer par un membre de la famille installé au Canada ${ }^{16}$. Les seules possibilités étaient de formuler une demande de résidence permanente, ou plus simple encore, demander un titre de séjour de courte durée.

L'entrée sur le territoire canadien de nos interlocutrices a suivi trois types de démarches. Tout d'abord, la majorité a demandé un visa de court séjour, principalement un visa de touriste, pour le Canada avec l'intention de déposer une demande d'asile une

15 Les demandeurs d'asile qui se présentent à la frontière sont dirigés vers l'une ou l'autre des provinces canadiennes selon plusieurs critères (maîtrise du français, membres de la famille installés au Canada, respect des niveaux d'immigration décidés par le gouvernement canadien, etc.). L'installation au Québec de ces derniers est régie par l'Accord Canada-Québec sur l'immigration et l'admission temporaire de ressortissants étrangers et la Loi sur l'immigration au Québec.

16 Le statut de résident permanent octroie la possibilité de faire une demande de parrainage pour un membre de sa famille (dispositif proche de celui du regroupement familial en France). Il existe également un parrainage dit privé : un groupe de personnes ou une association sont responsables du soutien financier de la ou des personnes réfugiées parrainées. 
fois au pays. Mais seules deux femmes ont réussi à obtenir ce visa. Les autres ont vu leur demande rejetée. D'autres, moins nombreuses, ont procédé à une demande de résidence permanente. Leurs demandes ont également été refusées, avant même l'examen médical obligatoire où leur séropositivité aurait pu constituer une difficulté supplémentaire. Dans ces cas de demande de visa de court ou long séjour, les refus étaient motivés par des « ressources financières insuffisantes durant le séjour» ou par un « manque de crédibilité dans les raisons de la venue ». Ces explications données par les agents consulaires ne font pas sens pour ces femmes qui proviennent de milieux plutôt aisés avec des garanties financières à la clé. Elles ont le sentiment qu'on a cherché à les empêcher de circuler :

« Je ne comprends pas pourquoi je n'ai pas eu le visa. Je suis pourtant propriétaire d'une maison, j'ai donné les titres de propriété à l'agent canadien. J'ai un travail de fonctionnaire très bien payé. Et on me refuse pourtant le droit de venir au

Canada » (Suzanne, trente-quatre ans, réfugiée du Burundi, arrivée en 2008).

Enfin, deux femmes ont été sélectionnées à l'étranger ${ }^{17}$ comme réfugiées par les programmes canadiens alors qu'elles avaient déposé une demande dans les pays africains où elles s'étaient provisoirement réfugiées, l'une au Kenya et l'autre en Afrique du Sud.

À cette étape de leur démarche, nos interlocutrices, qui pour la grande majorité connaissaient déjà leur statut sérologique, n'évoquent pas la possibilité d'un refus de titre permanent ou temporaire en raison de leur maladie. Pour les autres, qui ne feront le dépistage qu'une fois arrivées au Canada, le VIH n'est pas envisagé - même rétrospectivement - comme pouvant être un obstacle à l'obtention d'un titre de séjour de courte durée. Dans les deux cas, leur faible connaissance de la législation canadienne quant aux migrant-e-s malades, les conduit à penser que leur maladie ne peut constituer un motif de refus à leur demande. D'autant que, pour beaucoup d'entre elles, la contraction du VIH résulte des viols qu'elles ont subis, soit des violences sexistes qui peuvent paraitre constituer un motif légitime de demande d'asile si elles sont reconnues par la Commission de l'immigration et du statut de réfugié du Canada (CISR) comme motifs de persécution (nous reviendrons sur la question des motifs recevables pour déterminer de la nature et des motifs de la persécution).

Finalement, hormis quatre femmes dont nous venons de voir que deux avaient obtenu un visa temporaire et deux autres avaient été sélectionnées à l'étranger et obtenu ainsi la résidence permanente, toutes les autres candidates à l'exil se sont vues refuser un visa d'entrée au Canada. Découragées par la lenteur de l'instruction des demandes des titres de séjour et la lourdeur des garanties financières et des démarches, mais bien décidées à s'exiler au Canada, elles en sont venues à l'idée d'atteindre le pays par les États-Unis. Toutes ces femmes ont procédé de cette manière pour entrer au Canada. Elles ont mobilisé leurs réseaux proches ou éloignés - ceux de leur propre famille ou ceux de leur mari - pour demander aide et informations afin de mener à bien ce projet. C'est grâce à ces réseaux constitués de compatriotes, d'ami-e-s ou de connaissances ayant également fui le Rwanda et le Burundi après le génocide de 1993-1994, faisant office de conseillers et/ou intermédiaires, et qui vont leur rédiger des lettres d'invitation, qu'elles obtiennent

17 Les réfugiés réinstallés au Canada sont choisis à l'étranger et deviennent résidents permanents dès leur arrivée au Canada (Conseil canadien pour les réfugiés, http://ccrweb.ca/). 
sans difficulté un visa touristique d'entrée aux États-Unis. Il leur reste alors à se présenter au poste frontière du Canada et à déposer une demande d'asile. Elles ont majoritairement effectué le voyage, seules, bien que certaines aient été accompagnées de leur mari ou d'un enfant. Celles qui ont laissé derrière elles leur famille, ont dès le départ projeté un regroupement familial une fois leur titre de séjour obtenu. Cet éloignement a été déchirant, mais l'exil était l'unique voie pour garantir leur propre sécurité et par là même celle de leur famille :

«J'ai toujours fait passer les autres avant moi. Je sais que ça peut paraître égoïste que j'aie laissé mes enfants au pays. Mais je pense qu'au contraire c'est une façon de leur assurer un avenir. De toute manière les opposants politiques m'ont violée, ils voulaient me tuer. Leur mère serait probablement morte si j'étais restée » (Geneviève, quarante ans, femme réfugiée du Congo, arrivée en 2007).

Cette première partie de leur exil peut apparaître bien plus simple que pour nombre des émigrants d'Afrique subsaharienne, a fortiori des émigrantes (Laacher, 2010). Cependant, il faut souligner que le ressort de cette mobilisation, qui leur a facilité les modalités pratiques (administratives et financières) d'accès au territoire américain, repose sur un capital social et économique (Bourdieu, 1980) qu'elles détenaient bien avant l'émigration. Ces femmes à qui un visa canadien - y compris pour un séjour temporaire - avait été refusé, appartiennent dans leur pays aux classes sociales moyennes ou aisées et occupaient (elles ou leur mari) jusqu'au moment des massacres dans les années 1990, des positions avantageuses au sein des gouvernements ou des États rwandais et burundais. Positions que la plupart de ces femmes ont pu maintenir jusqu'à leur départ. Elles étaient et sont encore - en lien avec des personnes issues des mêmes milieux qui, elles aussi, ont été contraintes de quitter leur pays. Les coûts afférents à leur émigration sont très élevés (visas, billet d'avion, argent pour les dépenses une fois aux États-Unis et au Canada). Sans leur position sociale et leurs revenus, elles n'auraient pu faire face à la dépense.

\section{DROIT D'ASILE POUR LES FEMMES EXILÉES SÉROPOSI- TIVES}

Les parcours des femmes rencontrées présentent beaucoup de similitudes dans ce qu'elles vivent au cours des premiers jours de leur arrivée. Celles qui sont entrées par la frontière canado-américaine, où elles ont fait une demande d'asile auprès des agents frontaliers, ont été dirigées vers un centre d'hébergement montréalais où elles ont reçu des instructions pour se rendre au service du PRAIDA ${ }^{18}$ qui administre l'accueil, le soutien et l'information aux demandeurs d'asile.

18 Le Programme régional d'accueil et d'intégration des demandeurs d'asile (PRAIDA) offre plusieurs services - suivi psycho-social, accompagnement, référence, orientation, soutien financier, etc. - aux demandeurs d'asile et aux migrants en situation juridique précaire. Le mandat du PRAIDA couvre le territoire québécois en vertu de l'article 80 de la Loi sur la santé et les services sociaux (http://www.csssdelamontagne.qc.ca/services-du-praida/). 
Celles qui sont entrées avec un visa canadien de court séjour ont déposé leur demande d'asile ${ }^{19}$ auprès de la Commission d'immigration et du statut de réfugié (CISR) dans les jours qui suivent leur arrivée.

C'est à ce moment précis de la demande d'asile qu'entrent en jeu plusieurs acteurs, qui vont jouer un rôle primordial dans la construction de l'histoire de ces femmes devant " convaincre " le commissaire de la CISR de la véracité de leur persécution, d'abord sur dossier, puis plus tard, lors d'une l'audience. Les femmes rencontrées soulignent toutes que les associations montréalaises - de soutien aux immigrés et réfugiés ou aux personnes vivant avec le VIH-sida - et les intervenants du PRAIDA leur ont recommandé de demander l'aide d'un conseiller juridique (avocat ou consultant) pour préparer leur demande. C'est au cours de cette procédure que le VIH-sida devient un élément stratégique qu'il va falloir décider de taire ou de révéler, et dans ce cas mettre en forme, choix sur lequel va reposer « le montage » de la demande d'asile.

Les entretiens avec ces femmes réfugiées révèlent la manière dont la narration et la construction du récit sont contraints par les politiques migratoires envers les demandeurs d'asile vivant avec le VIH-sida. Elles doivent apprendre à jouer de ces contraintes, mais aussi des opportunités qui s'offrent dans ce cadre.

\section{Raconter les violences, mais en taire ses effets}

Le Canada fut, en 1993, le premier pays à se pourvoir de directives pour reconnaitre le statut de réfugiée aux femmes fuyant la persécution en raison de leur sexe (Wallace, 1996). Depuis, la reconnaissance de la violence fondée sur le sexe s'est relativement consolidée dans l'ensemble du système juridique canadien ${ }^{20}$. Ainsi, les violences - et parmi elles, les violences sexuelles - sont entendues comme pouvant ouvrir la voie à une demande d'asile, à condition que le lien soit établi entre le sexe, la persécution redoutée et un ou plusieurs des motifs de persécution mentionnés dans la Loi sur l'immigration (Directive 4, Commission de l'immigration et du statut de réfugié du Canada) ${ }^{21}$.

Pour autant, la Commission de l'immigration et du statut de réfugié du Canada (CISR) ne reconnaît pas a priori le lien entre les conditions vécues dans le pays d'origine et le risque de contamination au VIH ou, plus généralement, d'un état de santé dégradé même si la jurisprudence présente des cas où la maladie a pu influencer favorablement une décision des commissaires (Klein, 2001). Par ailleurs, la discrimination ne suffit pas à l'admissibilité au statut de réfugié. Pour que la demande d'asile d'une personne séropositive soit recevable, il faut démontrer la persécution vécue à raison d'au moins un des cinq motifs légaux (la race, la religion, la nationalité, l'opinion politique ou l'appartenance à un « groupe social ») ainsi que la non protection du pays d'origine. Les personnes vivant avec

19 Ce document est communément nommé FRP, le « Formulaire de renseignements personnels ». Il doit être retourné dans les vingt-huit jours après la date de réception du document par le réfugié. 20 http://ccrweb.ca/gendpersf.html

21 «Lorsqu'une femme affirme craindre d'être persécutée en raison de son sexe, il faut donc avant tout déterminer le lien entre le sexe, la persécution redoutée et l'un ou plusieurs des motifs de la définition (de réfugié) » (http://www.irb-cisr.gc.ca/fra/brdcom/references/pol/guidir/Pages/women. aspx). 
le VIH peuvent certes appuyer leur demande sur le fait d'appartenir à un « groupe social» dont la caractéristique est le VIH, caractéristique à raison de laquelle les membres sont individuellement et/ou collectivement victimes de persécution. D'ailleurs, la CISR a, par le passé, rendu des avis favorables à des demandes d'asile fondées sur une persécution à raison de l'appartenance à un groupe social défini par son statut sérologique (Klein, 2001 ; Barrère, 2006). Mais il s'avère, au vu de ce que les femmes nous ont raconté au cours des entretiens, qu'elles ont dû choisir entre dire leur état de santé ou bien dire les violences qu'elles avaient subies. Autrement dit, elles ont été prises dans un dilemme où, si elles optaient pour parler des violences, et notamment décrire les viols qu'elles avaient subis, elles devaient en taire les effets sur leur santé et leur pronostic vital.

Toutes les femmes que nous avons rencontrées ont estimé que leur vie était menacée d'abord en raison des violences - sexuelles, physiques et psychologiques -, menaces et intimidations dont elles faisaient l'objet et, secondairement, pour une minorité d'entre elles, à cause du VIH que ces violences avaient engendré, faute de suivi médical et d'accès aux médicaments. La violence se manifestait sous plusieurs formes, tant dans la sphère privée que dans la sphère publique. Elles parlent de violence conjugale (coups, rapports sexuels violents, contrôle de leurs sorties, violences psychologiques), de violences sexuelles perpétrées par d'autres hommes que leur conjoint, d'un climat plus général de tensions constantes, de peur de ce que demain apportera pour soi et sa famille.

Les femmes originaires du Rwanda et du Burundi ne font pas de distinction entre les violences commises lors du génocide - la plupart ont été violées, par des soldats ou des membres de leur entourage - celles perpétrées après ou celles ayant lieu dans la sphère privée. C'est précisément ce climat de répétitions des violences, caractérisé par la menace constante d'un basculement vers les agressions, qui leur a rendu la vie insupportable :

«Le retour en 1999 [elle s'était réfugiée en 1994 dans un pays limitrophe au Burundi] au Burundi, c'était comme si je revivais le passé. J'avais vécu des moments très difficiles en 1994, c'était en pleine guerre et j'ai été violée. Et puis en revenant, j'ai revécu les mêmes sévices. Là, j'ai dit trop c'est trop. [...] J'ai craqué. J'ai dit, soit je me suicide, soit je quitte le pays » (Lucille, cinquante ans, réfugiée du Burundi, arrivée en 2000).

«Mon mari me battait avant la guerre. Il me violait aussi parfois et c'était pire après le début des conflits. Et puis un soir, des soldats sont entrés chez nous. Ils m'ont violée devant les enfants. Mon mari était en brousse pour se cacher. Là, c'était la catastrophe. J'avais l'impression que j'étais entourée de bêtes. Les hommes, c'étaient des bêtes. Ils voulaient me rendre faible, me faire du mal. C'était la même chose. C'est une arme contre les femmes » (Claire, quarante-neuf ans, réfugiée du Burundi, arrivée en 2005).

Elles soulignent ainsi le fait que les mécanismes d'inégalités entre les femmes et les hommes se perpétuent aussi hors des périodes de conflit. En cela, elles montrent comment ces violences ne "résultent pas de comportements individuels isolés et atypiques, voire "aberrants", mais reflètent au contraire des structures et des normes sociales profondément inégalitaires » (Freedman et Valluy, 2007 : 13). Elles ont toutes pressenti que rester dans leur pays d'origine les priverait d'avenir et les laisseraient mourir dans la crainte constante des agressions physiques et/ou sexuelles. 
Au moment de l'exil, celles qui connaissaient leur séropositivité ont pris dans leurs bagages des ordonnances de leur médecin et, si possible, une réserve d'antirétroviraux. Mais, lors de la formulation de leur demande d'asile, les réfugiées interviewées ont plutôt cherché à taire leur maladie. Selon leurs dires unanimes, ces femmes ont été dissuadées d'axer leur demande sur la maladie alors que certaines d'entre elles établissaient un lien direct entre leur contamination au VIH et les violences sexuelles subies.

"Quand j'ai raconté mon histoire à l'avocat, forcément j'ai parlé du sida. On m'a contaminée quand j'ai été emprisonnée au Congo et violée par les geôliers. J'avais passé un test comme mon mari avant de me marier et c'était négatif. Quand j'ai appris que j'étais malade, c'était au Canada. Mais là, l'avocat a dit de ne pas en parler, que de toute manière c'était inscrit dans mon dossier. Pour moi, c'est lié aux multiples viols, mais je ne peux pas en parler devant le commissaire de l'immigration » (Geneviève, quarante ans, réfugiée du Congo, arrivée en 2007).

Cette «stratégie » nous est confirmée par une intervenante œuvrant au sein d'une association pour femmes séropositives montréalaise qui souligne que les femmes prendraient trop de risques à mettre en avant leur séropositivité au cours des audiences de la CISR, quand parler de la violence qu'elles ont subie en tant que femme suffit à nourrir leur argumentaire :

«Les femmes que l'on accueille ici ne savent pas comment fonctionne le système et elles parlent généralement sans trop de tabous de leur maladie. Mais, au Canada, pour avoir le statut de réfugié, la maladie elles ne peuvent pas en parler sinon le juge à la commission risque de penser qu'elles sont là pour avoir des traitements.

Mais tout ça il faut leur dire, elles doivent parler des violences dont elles ont été victimes dans leur pays » (Intervenante, association montréalaise de support aux femmes séropositives).

Nous retrouvons également dans les récits des femmes des propos congruents qui soulignent leur apprentissage de ce qu'il faut taire et de ce qu'il faut révéler dans leur demande d'asile :

"Les avocats et les associations m'ont très vite dit de parler de ce que j'ai vécu, de la violence subie parce que j'étais une femme. La maladie, on apprend très vite à ne pas en parler, sauf au médecin » (Antoinette, trente-six ans, femme réfugiée du Burundi, arrivée en 2007).

Les lois canadiennes qui régissent l'immigration - relayées par l'encadrement des associations et des avocats - conduisent les femmes à intérioriser l'image attendue d'elle : celle de la «bonne » réfugiée dont le corps persécuté et violenté du fait de leur statut de femme ouvre le droit à une demande d'asile, et non pas le corps malade, vu comme un fardeau financier. Quoique certaines aient voulu mettre en avant le VIH et montrer que, justement, il est une des conséquences des violences sexuelles subies, elles ont fini par se résigner à se ranger aux arguments stratégiques de leurs conseillers juridiques. Finalement, toutes celles qui sont passées devant le commissaire de la CISR, ont 
fondé leurs demandes d'asile sur les persécutions genrées des femmes ${ }^{22}$, contraintes d'en taire les effets sur leur santé, dont la séropositivité au VIH qu'elles ont à assumer depuis. Paradoxalement, leur expérience de la demande d'asile au Canada, leur permet d'être reconnues pour les persécutions qu'elles ont subies comme femmes, tout en les amputant de la reconnaissance de leur statut de malades victimes de cette même violence.

Le travail des associations, des conseillers juridiques et des réseaux sociaux (entourage de proximité et notamment des personnes séropositives rencontrées dans les associations) conduit ces femmes à incorporer les règles législatives canadiennes, en tant que système à la fois habilitant et contraignant (Giddens, 2005). Les politiques canadiennes - par les conditions qu'elles édictent d'accès restrictif au territoire, au séjour et aux soins - imposent des choix aux femmes violentées et devenues séropositives et, de ce fait, influencent leurs parcours migratoires et thérapeutiques. Quoi qu'il en soit, en choisissant l'exil ces femmes sont redevenues actrices de leur vie, ce dont elles n'étaient plus sûres dans leur pays. Face à ces lois, les femmes continuent d'agir en actrices qui disposent de « capacité d'accomplir des choses » (Giddens, 2005 : 345), même au prix d'une stratégie qui axe leur demande d'asile sur les violences en éclipsant le VIH. Mais, au-delà de l'accès au territoire, se pose pour ces femmes malades la question de l'accès aux soins, notamment aux ARV pour les plus vulnérables à l'évolution de l'immunodépression que créé le VIH. Au côté de la politique d'accès au titre de séjour, c'est bien tout le système qui organise l'intégration des réfugiés dans la société canadienne qui structure les trajectoires des femmes rencontrées et ce, dans un processus dynamique où, comme actrices habilitées, elles développent des stratégies et saisissent des ressources leur permettant d'en appréhender les rouages.

\section{RÉFUGIÉES SÉROPOSITIVES : LE POIDS DU SOUPÇON SUR LA TRAJECTOIRE DES FEMMES}

Les femmes interrogées ne rapportent pas de problème majeur dans l'accès à une couverture de santé lors de leur arrivée et dans les mois qui suivent leur installation au Canada.

Suite à leur première rencontre avec des intervenants du PRAIDA, elles sont dirigées vers un médecin désigné comme expert par les autorités canadiennes, qui procèdera à un bilan général de leur santé au cours duquel il leur sera fait une prise de sang. C'est lors d'un second rendez-vous que ce médecin leur communique les résultats de ce bilan et leur annonce, le cas échéant, leur séropositivité au VIH. L'annonce est faite avec d'autant moins de ménagement que les femmes concernées sont plus ou moins soupçonnées, semble-t-il, d'avoir usé de la demande d'asile pour venir se faire soigner au Canada. C'est le cas de Nicole, à qui il est reproché de ne pas avoir révélé sa séropositivité - qu'elle ignorait en réalité -, lors de son arrivée sur le territoire canadien :

22 Persécutions que Freedman et Valluy définissent comme : « les persécutions des femmes parce $q u$ 'elles sont des femmes, mais aussi les persécutions qu'elles subissent comme femmes, c'està-dire comme acteurs sociaux tributaires d'un rôle social et d'une position voire d'une fonction, imputés à la "femme" dans la société de référence » (Freedman et Valluy, 2007 : 10). 
« Je n'ai pas aimé la façon dont le médecin de l'immigration m'a annoncé la nouvelle. J'ai failli m'évanouir. Il m'a dit: "pourquoi tu as menti ? Pourquoi tu n'as pas dit la vérité?" Je lui ai répondu: "quelle vérité ?" Il m'a dit: "que tu étais séropositive?" Je lui ai dit qu'il me l'annonçait. Il m'accusait d'avoir menti à l'immigration. J'étais en colère contre lui, mais je me suis retenue et je suis restée polie.

Il m'a dit d'aller consulter un autre médecin, je suis partie. Mais j'étais tellement mal et en colère de me faire traiter de menteuse que ça m'a pris plusieurs semaines avant d'aller voir l'autre médecin » (Nicole, quarante-neuf ans, réfugiée du Burundi, arrivée en 2004).

Cette interaction difficile avec le médecin mandaté par le ministère canadien de l'Immigration, n'est pas l'unique occasion où les femmes rapportent avoir été apostrophées sur les raisons de leur asile au Canada et où il leur a été fait part d'une certaine suspicion quant au motif réel de leur venue :

«Le juge à la commission des réfugiés m'avait demandé si j'étais venue au Canada pour me faire soigner... J'étais complètement choquée. Après tout ce que j'ai vécu, les violences, les viols et tout... Je ne comprenais pas pourquoi il pouvait penser cela. J'avais l'impression qu'il mettait de côté ce que je lui racontais pour ne penser qu'à la maladie et que je venais ici pour avoir des médicaments » (Ornella, quarante-quatre ans, femme réfugiée du Burundi, arrivée en 2007).

Or, ce préjugé est non seulement infondé, mais il fait fi des violences que ces femmes ont subies. À travers cette accusation de chercher à taire ce qui serait supposément le «véritable motif de leur venue », ces femmes font une nouvelle fois l'expérience d'une disqualification de leur parole. Une censure de leur vécu qu'elles ressentent comme un soupçon profondément injuste quant au poids des facteurs politiques qui ont conduit à leur exil. Un sentiment de malaise intolérable face à l'image qu'on leur renvoie, bâtie sur l'idée qu'elles ne seraient venues au Canada que pour la prise en charge de leur maladie par le système de santé. Ces situations ont des impacts avant tout d'ordre psychologique le repli sur soi, la perte de confiance en soi - mais aussi d'ordre relationnel avec la société canadienne dans son ensemble, par la méfiance face aux professionnels de santé et aux représentants du gouvernement qu'elles suscitent.

Par ailleurs, les pratiques de ces médecins à leur endroit induisent un véritable choc, car plusieurs d'entre elles ont découvert abruptement qu'elles étaient contaminées. Un choc d'autant plus violent que le médecin n'a pas pris la peine de les informer du dépistage. Plusieurs femmes témoignent qu'elles n'ont pas été informées qu'elles seraient soumises au dépistage du VIH et n'ont donc pas été préparées à un résultat positif. Pourtant, les normes professionnelles en vigueur recommandent que le dépistage du VIH soit précédé et suivi d'un entretien de counselling ${ }^{23}$ avec le patient (Réseau juridique canadien $\mathrm{VIH} /$ sida, 2011). Un choc encore aggravé par le ton incriminant que peuvent prendre les professionnels de santé, un choc qui les traumatise et les sidère au point de retarder l'accès à des soins pourtant urgents, comme le raconte Adeline au sujet du jugement sans appel du médecin sur ses supposés « comportements sexuels inconsidérés ». Ou comme dans le cas de Geneviève qui aurait dû se protéger quand elle a été violée :

23 Consultation axée sur l'aide à la résolution de problèmes médicaux, sociaux et/ou psychologiques, grâce aux conseils, à l'écoute et l'échange avec un professionnel de santé. 
"Quand le médecin m'a donné les résultats, il m'a dit que je ne savais pas comment me protéger dans mes relations sexuelles. Je lui ai demandé de s'asseoir calmement et parler. Il m'a dit qu'il n'avait pas le temps, qu'il avait beaucoup de patients à voir. Je n'ai pas du tout aimé sa façon de faire. Je suis restée dix minutes dans son bureau quand il est parti. Je ne bougeais pas. J'étais étourdie. Il n'était pas humain et il m'a dit d'aller au plus vite dans un autre hôpital. Mais j'ai passé un mois avant d'y aller. Je n'en revenais pas. Je ne pouvais pas imaginer avoir cette maladie... » (Geneviève, quarante ans, réfugiée du Congo, arrivée en 2007).

L'absence d'information sur le dépistage, la privation d'entretien de counselling, les révélations de diagnostics sans précaution, voire brutales, sans complément d'information sur la maladie ou son suivi que nous rapportent ces femmes, sont autant d'exemples de défaillance que confirment d'autres études (Bisaillon, 2010 ; Réseau juridique canadien VIH/sida, 2011).

Du fait de leur statut de demandeuses d'asile, elles accèdent aux soins par le biais du Programme fédéral de santé intérimaire (PFSI) ${ }^{24}$, un système d'assistance qui les couvrira le temps d'instruction de leur dossier ${ }^{25}$. Ce programme leur assure un suivi médico-psycho-social, leur offrant avant tout une couverture de base des soins de santé et des services sociaux essentiels et urgents (services médicaux et hospitaliers, examens, tests de laboratoire, actes de chirurgie, médicaments de base sur ordonnance, etc.). Les demandeurs d'asile séropositifs sont, à ce titre, médicalement pris en charge et reçoivent leur traitement gratuitement pendant la durée de leur couverture par le PFSI. Cependant, les soins ne peuvent être dispensés que par des professionnels dans des établissements de santé mandatés par le gouvernement fédéral dans le cadre de ce programme. Cette contrainte peut complexifier le recours aux soins et occasionner encore des retards, en particulier dans les moments où les femmes sont le plus fragilisées par la maladie et la situation migratoire, leurs lieux de résidence n'étant pas forcément à proximité des centres de soins accessibles aux requérants :

«J'ai voulu une fois aller dans le centre de santé à côté de chez moi parce que je n'arrêtais pas de saigner. Et là-bas, on me dit que mon papier de réfugié ce n'est pas bon: "on ne prend pas ça ici, on prend seulement la carte d'assurance maladie!' Je suis allée là parce que je n'arriverais pas à aller à l'hôpital. J'ai demandé à une cousine de venir m'aider et elle est venue, on est parties à l'hôpital et j'ai eu une transfusion. Selon moi, on m'a refusé des soins parce que je n'ai pas la carte d'assurance maladie, mais un papier de réfugiée » (Jeannette, quarante ans, réfugiée du Burundi, arrivée en 2007).

Dans l'ensemble les femmes disent avoir reçu des soins adéquats et ne pas avoir eu à débourser d'argent pour obtenir une consultation médicale ou des traitements. Mais beaucoup d'entre elles soulignent aussi que la prise en charge médicale et sociale

24 D'autres catégories sont admissibles : les réfugiés sélectionnés à l'étranger en cas de retard dans l'obtention de leur droit au régime d'assurance maladie et les autres personnes à protéger (victimes de traite, personnes sous moratoire, etc.).

25 En cas de refus de la demande d'asile, cette couverture de santé est prolongée aussi longtemps que durent les procédures de recours. Il est suspendu en cas de rejet et le moment où les demandeurs déboutés « tombent » dans la catégorie des sans-papiers. 
associée à leur statut juridique est limitée et contraignante. Certaines limites émergent très vite, avec la non-reconnaissance des droits accordés aux bénéficiaires du PFSI dans de nombreux établissements de santé et un panier de soins couverts somme toute assez réduit. Nos analyses rejoignent ainsi plusieurs études qui soulignent les dysfonctionnements, dans l'accès aux soins pour les réfugiés au Canada, et en rapportent l'impact négatif sur la santé - physique et psychologique - des patient-e-s (Elgersma, 2008 ; Gagnon, 2002 ; Oxman-Martinez et al., 2005 ; Rousseau et al., 2008 ; Ter Kuile, 2007).

\section{CONCLUSION}

Nous avons voulu mettre en évidence la manière dont le cadre juridique et administratif canadien influence le choix des motifs que les femmes peuvent invoquer pour déposer une demande de séjour : «Ces dispositions, ainsi que d'autres facteurs structurels, façonnent dans une certaine mesure les stratégies des migrant·e's, qui orientent leur parcours en fonction des opportunités, utilisent ou contournent les dispositifs existants » (Lesselier, 2008 : 193). Les femmes rencontrées butent d'abord sur l'impossibilité d'obtenir le droit d'entrer sur le territoire canadien, puis elles mobilisent les ressources de leur capital social pour obtenir un titre de séjour pour les États-Unis, ce qui leur permet de demander l'asile à la frontière canado-américaine. Bien qu'ayant vécu des violences aux formes multiples, l'exil leur permet de recouvrer une certaine maîtrise de leur vie à travers la demande d'asile. Elles y trouvent un cadre où leur parole sur les violences faites aux femmes, auparavant indicible ou, à tout le moins inaudible, est non seulement entendue, mais reconnue puisque plus de la moitié des femmes interrogées obtiennent le statut de réfugiées, les autres étant en attente d'une décision de la CISR. Pour elles, il s'agit de dénoncer les persécutions dont elles ont été victimes et de reprendre confiance dans un cadre normatif qui affirme l'illégitimité des violences subies et leur offre une protection étatique via le statut de réfugiée. Cette reconnaissance des violences subies leur permettra d'envisager une nouvelle vie où leur séropositivité au VIH sera prise en charge.

Les récits de ces femmes montrent la nécessité de sortir de la dichotomie entre sphères privée et publique dans l'analyse des violences. Leurs propos dénaturalisent les violences faites aux femmes et soulignent la gravité des actes commis, qu'ils se soient déroulés lors de situations de violences extrêmes (génocide, désorganisation étatique, guerre civile, etc.) ou qu'ils aient été commis dans le cours de la vie conjugale. Dans les deux cas, ces violences produisent un effet de déshumanisation du sujet, un effet que ces femmes réussissent à surmonter, d'abord en y mettant un terme et en fuyant leur pays, puis en revendiquant le statut de réfugiée sur la base de persécutions liées au genre. Cependant, si le droit au séjour pour ces migrantes est accessible à raison des violences et des persécutions genrées subies, il ne l'est pas du fait d'une maladie nécessitant des soins et des traitements déficitaires dans leur pays d'origine. Parce que les politiques migratoires canadiennes tendent à caractériser le VIH comme un « fardeau économique » et non comme un révélateur d'inégalités, les migrantes sont souvent amenées à taire leur maladie et à faire face au soupçon sur les véritables motifs de leur demande d'asile au Canada.

Le projet de loi C-31 déposé le 16 février 2012 par le ministre de la Citoyenneté, de l'Immigration et du Multiculturalisme visant à réformer le système canadien d'octroi 
de l'asile, qui entrera en vigueur le 29 juin $2012^{26}$, contient des changements législatifs préoccupants pour les femmes réfugiées. Notamment, les délais raccourcis pour la présentation d'une demande d'asile après l'entrée au Canada (passant de vingt-huit jours à quinze jours) permettront difficilement une préparation adéquate des femmes victimes de violences pour qui la création d'une relation de confiance avec un conseiller juridique est essentielle afin de réussir à raconter leur histoire. Qui plus est, la volonté d'instaurer une liste de "pays d'origine désignés » $(\mathrm{POD})^{27}$ occulte la variété des persécutions genrées vécues par les femmes dans les pays désignés comme "sûrs » qui surviennent principalement dans la sphère privée, traditionnellement à l'abri de la surveillance des États (Devidal, 2007 : 440). Ces changements fondamentaux du système d'asile font craindre le recul du Canada en matière de protection des réfugiés et sont particulièrement préjudiciables pour les femmes en quête de reconnaissance de persécutions liées au genre.

\section{Références bibliographiques}

ALI Jennifer S., McDERMOTT Sarah and GRAVEL Ronald G. (2004) Recent Research on Immigrant Health from Statistics Canada's Population Surveys, Canadian Journal of Public Health, 95 (3), pp. 1-9.

BARRÈRE Graciela (2006) La santé et le droit de l'immigration : une perspective comparative du Canada et de la France, Mémoire de droit non publié, Université de Montréal, 145 p.

BEISER Morton, HOU Feng, HYMAN Ilene et TOUSIGNANT Michel (1998) Étude des nouveaux enfants immigrants qui grandissent en tant que Canadiens, Ottawa-Hull, Direction générale de la recherche appliquée.

BISAILLON Laura M. (2010) Human rights consequences of mandatory HIV screening policy of newcomers to Canada, Health and Human Rights: An International Journal, 12 (2), pp. 119-134.

BOURDIEU Pierre (1980) Le capital social, Actes de la recherche en sciences sociales, 31, p. 2.

CHEN Jiajian, NG Edward and WILKINS Russell (1996) The health of Canada's immigrants in 1994-95, Health Reports, 7 (4), pp. 33-45.

CITOYENNETÉ ET IMMIGRATION CANADA (CIC), [en ligne]. URL : http://www.cic.gc.ca/ francais/index.asp

COGNET Marguerite (2004) La vulnérabilité des immigrés : Analyse d'une construction sociale, in Francine Saillant, Michèle Clément et Charles Gaucher Dirs., Identités Vulnérabilité, Communautés, Québec, Éditions Nota Bene, pp. 155-188.

DE MAIO Fernando G. (2010) Immigration as pathogenic: A systematic review of the health of immigrants to Canada, International Journal for Equity in Health, 9 (27), [en ligne]. URL : http://www.equityhealthj.com/content/pdf/1475-9276-9-27.pdf

DE MAIO Fernando G. and KEMP Eagan (2010) The deterioration of health status among immigrants to Canada, Global Public Health, 5 (5), pp. 462-478.

DEVIDAL Pierrick (2007) Pour un système de protection active des femmes réfugiées, in Jane Freedman et Jérôme Valluy Dirs., Persécutions des femmes. Savoirs, mobilisations et protections, Broissieux, Éditions du Croquant, pp. 431-450.

ELGERSMA Sandra (2008) Immigration status and legal entitlement to insured health service, Ottawa, Library of Parliament, Political and Social Affairs Division, 11 p.

26 http://www.irb-cisr.gc.ca/Fra/media/newsnouv/2011/Pages/reform1108.aspx

27 Pays désignés par le gouvernement canadien comme sûrs, démocratiques et dont leurs ressortissants déposent peu de demandes d'asile au Canada (http://www.cic.gc.ca/francais/refugies/ reforme-surs.asp). 
FREEDMAN Jane et VALLUY Jérôme (2007) Introduction. Persécutions genrées des femmes, in Jane Freedman et Jérôme Valluy Dirs., Persécutions des femmes. Savoirs, mobilisations et protections, Broissieux, Éditions du Croquant, pp. 7-32.

GAGNON Anita J. (2002) The Responsiveness of the Canadian Health Care System Towards Newcomers, Ottawa, Royal Commission on the Future of Health Care in Canada, Health Canada, $45 \mathrm{p}$.

GIDDENS Anthony (2005) La constitution de la société. Éléments de la théorie de la structuration (traduit par M. Audet), Paris, Presses Universitaires de France, 474 p.

GUILLOU Anne-Yvonne (2009) Immigration thérapeutique, immigration pathogène. Abandonner le « parcours thérapeutique » pour l'expérience migratoire (Commentaire), Sciences sociales et santé, 27 (1), pp. 63-71.

HYMAN Ilene (2009) Racism as a determinant of immigrant health, Ottawa, Strategic Initiatives and Innovations Directorate of the Public Health Agency of Canada, $19 \mathrm{p}$.

JOLIVET Violaine (2007) La notion de trajectoire en géographie, une clé pour analyser les mobilités ? Regard croisé sur des trajectoires caribéennes. EchoGéo, 2, [en ligne]. URL : http:// echogeo.revues.org/1704

KLEIN Alana (2001) Immigration et VIH/sida: rapport final, Réseau juridique canadien VIH/sida, $106 \mathrm{p}$.

KIRMAYER Laurence J., NARASIAH Lavanya, MUNOZ Marie, RASHID Med, RYDER Andrew G., GUZDER Jaswant, HASSAN Ghayda, ROUSSEAU Cécile and POTTIE Kevin (2011) Common mental health problems in immigrants and refugees: general approach in primary care, Canadian Medical Association Journal, 183 (12), pp. 959-967.

LAACHER Smaïn (2010) De la violence à la persécution, femmes sur la route de l'exil, La Dispute, $169 \mathrm{p}$.

LAROCHE Mireille (2000) Health Status and Health Services Utilization of Canada's Immigrant and Non-Immigrant Populations, Canadian public policy - Analyse de politiques, 26 (1), pp. 51-75.

LESSELIER Claudie (2008) Politiques d'immigration en France : Appréhender la dimension du genre, Femmes, genre, migrations et mondialisation : un état des problématiques, Cahiers du CEDREF, pp. 189-208.

McDONALD James Ted and KENNEDY Steven (2004) Insights into the "healthy immigrant effect": Health status and health service use of immigrants to Canada, Social Science \& Medicine, 59, pp. 1613-1627.

NEWBOLD Bruce and DANFORTH Jeff (2003) Health status and Canada's immigrant population, Social Science \& Medicine, 57 (10), pp. 1981-1995.

McKEARY Marie and NEWBOLD Bruce (2010) Barriers to Care: The Challenges for Canadian Refugees and their Health Care Providers, Journal of Refugee Studies, 23 (4), pp. 523-545.

MOREAU Sylvie, ROUSSEAU Cécile et MEKKI-BERRADA Abdelwahed (1999) Politiques d'immigration et santé mentale des réfugiés : profil et impact des séparations familiales, Nouvelles pratiques sociales, 12 (1), pp. 177-196.

OXMAN-MARTINEZ Jacqueline, HANLEY Jill, LACH Lucyna, KHANLOU Nazilla, WEERASINGHE Swarna and AGNEW Vijay (2005) Intersection of Canadian Policy Parameters Affecting Women with Precarious Immigration Status: A Baseline for Understanding Barriers to Health, Journal of Immigrant Health, 7 (4), pp. 247-258.

RÉSEAU JURIDIQUE CANADIEN VIH/SIDA (2011) Les politiques canadiennes sur l'immigration et leur impact sur les personnes vivant avec le VIH, Feuillet d'information, [en ligne]. URL : http://www.aidslaw.ca/publications/publicationsdocFR.php?ref=98

ROUSSEAU Cécile, TER KUILE Sonia, MUNOZ Marie, NADEAU Lucie, OUIMET Marie-Jo, KIRMAYER Laurence and CRÉPEAU François (2008) Health care access for refugees and immigrants with precarious status: Public health and human right challenges, Canadian Journal of Public Health, 99 (4), pp. 290-292. 
SPIJKERBOER Thomas (2000) Gender and Refugee Status, Aldershot, Ashgate Dartmouth, 286 p. TER KUILE Sonia, ROUSSEAU Cécile, MUNOZ Marie, NADEAU Lucie and OUIMET Marie-Jo (2007) The Universality of the Canadian Health Care System in Question: Barriers to Services for Immigrants and Refugees, International Journal of Migration, Health, and Social Care, 3 (1), pp. 15-26.

WALLACE Rebecca (1996) Making the Refugee Convention Gender Sensitive: The Canadian Guidelines, International \& Comparative Law Quarterly, 45 (3), pp. 702-711.

WEIBE Sarah (2009) Producing Bodies and Borders: A Review of Immigrant Medical Examinations in Canada, Surveillance \& Society, 6 (2), pp. 128-141.

ZHAO Jun, XUE Li and GILKINSON Tara (2010) Health Status and Social Capital of Recent Immigrants in Canada. Evidence from the Longitudinal Survey of Immigrants to Canada, Ottawa, Citizenship and Immigration Canada, $25 \mathrm{p}$. 


\section{Femmes africaines séropositives en quête d'asile. Opportunités et contraintes de la politique migratoire canadienne Émilie ADAM-VÉZINA}

Cet article explore certains aspects des trajectoires d'exil de femmes réfugiées de la Région des Grands Lacs africains vers le Canada et atteintes du VIH-sida. Si les facteurs pré et post migratoires peuvent avoir un impact sur leurs conditions de vie et leur santé, l'angle que nous avons privilégié ici traite des effets des conditions législatives d'accès au territoire et aux soins pour ces migrantes. Après un bref exposé du contexte législatif canadien qui régit l'entrée sur le territoire et des dispositions relatives aux migrants malades et en particulier aux personnes séropositives au VIH nous montrerons, au travers des récits des femmes rencontrées, fuyant principalement des violences et persécutions genrées, comment ces politiques influencent leur trajectoire et leur santé. Nous verrons que la législation canadienne pose un ensemble de contraintes pour les réfugiées séropositives, tout en constituant une ressource mobilisable propre fournissant certaines opportunités.

\section{HIV-positive African Women in Search of Asylum. Opportunities and Constraints of the Canadian Migratory Policy Émilie ADAM-VÉZINA}

This article explores certain aspects of the exile trajectories of women living with HIV-AIDS from the African Great Lakes and coming to Canada. If pre- and post-migratory factors can have an impact on their living conditions and health, we will focus rather on the effects of Canadian legislation on access to the territory and to healthcare for these migrants. After a brief summary of Canadian legislation governing the entry for sick migrant and especially HIV-positive migrants we will show through the stories of the women interviewed, who mainly fleeing violence and persecution gendered, how these policies affect their trajectory and their health. We will see that Canadian law is a set of constraints for refugees with HIV, while providing a resource available to capture some specific opportunities.

\section{Mujeres africanas seropositivas en busca de asilo. Oportunidades y limitaciones de la política migratoria canadiense Émilie ADAM-VÉZINA}

Este artículo trata de las trayectorias de exilio de mujeres de origen africana (región de los Grandes Lagos) y refugiadas en Canadá. Más precisamente, el artículo se centra sobre el caso de mujeres viviendo con el VIH-SIDA. Si los factores ante- y post-migración puedan afectar a sus vidas y su salud, este articulo pone de manifiesto los efectos del acceso al territorio y a los servicios de salud. Después de una síntesis de la legislación canadiense sobre la entrada y las disposiciones para los pacientes migrantes - y especialmente para las personas VIH-positivas -, este artículo muestra, a través de las historias de vida de las mujeres encontradas, cómo estas políticas afectan a su trayectoria y su salud. Veremos que la legislación canadiense impone un conjunto de restricciones para estas mujeres que viven con el VIH, mientras que puede ser un recurso para aprovechar algunas oportunidades específicas. 